\title{
Experimental studies on two-phase flow patterns aboard the Mir space station
}

\author{
J.F. Zhao a, ${ }^{\text {, }}$, J.C. Xie ${ }^{\text {a }}$, H. Lin ${ }^{\text {a }}$, W.R. Hu ${ }^{\text {a }}$, A.V. Ivanov ${ }^{\text {b }, ~ A . Y u . ~ B e l y a e v ~}{ }^{b}$ \\ ${ }^{a}$ National Microgravity Laboratory, Institute of Mechanics, Chinese Academy of Sciences, \\ 15 Zhong Guan Cun Road, Beijing 100080, China \\ ${ }^{\mathrm{b}}$ Keldysh Research Center, Federal State Unitary Enterprise, Russian Aviation and Space Agency, \\ 8/10 Onezhskaya, Moscow 125438, Russia
}

Received 29 June 2000; received in revised form 20 May 2001

\begin{abstract}
A series of experiments on two-phase gas-liquid flow patterns in a test tube with a length of $356 \mathrm{~mm}$ and an inside diameter of $10 \mathrm{~mm}$ were performed aboard the Mir Space Station in August 1999. Carbogal and air were used as the liquid and the gas phase, respectively. In the present paper, the experimental results at the background microgravity environment of the Mir Space Station (no more than $10^{-5} \mathrm{~g}$ ) were reported. Five kinds of flow patterns, namely dispersed bubble flow, bubble flow, slug flow, slug-annular transitional flow, and annular flow, were observed in the space experiment. Due to the small length-to-diameter ratio of the test tube used in the present study, the observed flow patterns should be considered to be developing ones. The experimental results were compared with the model proposed previously which accounts for the entrance effects on the flow pattern transitions. A good agreement between the predictions and the experimental data was obtained. Some widely used models developed based on the analysis of fully developed two-phase flow at microgravity were also compared with the present data in order to make evaluations of these models and to have some insights on the flow evolution. (C) 2001 Elsevier Science Ltd. All rights reserved.
\end{abstract}

Keywords: Microgravity; Two-phase flow; Flow pattern; Flow pattern transition; The Mir Space Station

\section{Introduction}

Two-phase gas-liquid flow has wide applications both on Earth and in space. It occurs in a variety of process equipments on Earth, such as petroleum production facilities, condensers and

\footnotetext{
${ }^{*}$ Corresponding author. Fax: +86-10-62615524.

E-mail address: jfzhao@imech.ac.cn (J.F. Zhao).
} 
re-boilers, power systems and core cooling of nuclear power plants during emergency operation. The space applications include wastewater reclamation units, active thermal control systems, power cycles, storage and transfer of cryogenic fluids, and so on.

For conditions of technological interest, there are several major types of the phase distributions, i.e. flow patterns, observed for two-phase flows in pipes. Characteristics of the flow patterns and the conditions under which they exist depend on many factors, such as the orientation of the pipe with respect to gravity, the flow rates of the gas and liquid phases, the pipe diameter, the system pressure, and so on. Among them the gravity influence on two-phase flow patterns is a dominant and complicated factor at normal gravity, resulting in a variety of perturbations, instabilities, and generally undesirable unsteady features. As a result, our present knowledge on the mechanism controlling the flow pattern transitions, which is built on a foundation of a large empirical database developed with the aid of numerous meticulous experiments, is far from complete.

At low- and microgravity conditions, due to the weakening or removal of the gravity, twophase flows are essentially much simpler than those at normal gravity. Therefore, the investigations of two-phase flows at low- and microgravity conditions will be conducive to revealing the mechanism underlying the flow pattern transitions, and then developing more accurate models for the flow pattern transitions of two-phase flows both on Earth and in space.

In the past one and a half decades, some experimental investigations of the two-phase flows have been conducted in the reduced gravity conditions aboard an aircraft flying parabolic trajectories (Dukler et al., 1988; Colin et al., 1991; Zhao and Rezkallah, 1993, 1995; Bousman et al., 1996; Lowe and Rezkallah, 1999; Zhao et al., 2000). However, there exist some drawbacks to this method. For example, the overload of near $2 g$ before and after each reduced gravity portion and the rapid change in gravitational fields can induce undesired system transients. Unsteady residual gravity acceleration, typically in the level of $\left(10^{-1}-10^{-3}\right) g$, that depends on the pilot, the weather and other uncontrolled factors is another disadvantage. Free falls in a drop tower are also used to produce a short microgravity period (less than $10 \mathrm{~s}$, typically 2-5 s) for the studying of microgravity two-phase flows (Dukler et al., 1988; Wölk et al., 1999). The limited test period associated with this method makes it difficult to eliminate the transient effect of the rapid change in gravitational fields. There is also an experiment on two-phase pressure drop at long-term, steady microgravity condition aboard the US Space Shuttle (Reinarts et al., 1995). However, no visual observation of the flows was made in their experiment.

Some other creative ways for simulating two-phase flows at microgravity condition in a laboratory environment, such as the use of small inside diameter tubes (Galbiati and Andreini, 1994) and density-matched immiscible liquids (Lovell, 1985), were also used to increase the impact of surface tension force and reduce that of gravity. These methods can create gravity-independent two-phase flows with some characteristics of two-phase flows at reduced gravity. The disadvantage, however, is that they are not complete simulations of microgravity two-phase gas-liquid flows.

Efforts have also been made to develop the criteria of the flow pattern transitions at reduced gravity (Lee, 1987, 1993; Dukler et al., 1988; Colin et al., 1991, 1996; Zhao and Rezkallah, 1993; Carron and Best, 1994, 1996; Rezkallah and Zhao, 1995; Reinarts, 1995; Bousman et al., 1996; Rezkallah, 1996; Jayawardena et al., 1997; Lowe and Rezkallah, 1999; Zhao and Hu, 2000). 
A comprehensive review of the different methods of testing and modelling of the flow pattern transitions at reduced gravity can be found in Zhao (1999).

A series of two-phase flow experiments were performed aboard the Russian Mir Space Station in August 1999. The long-term, steady microgravity condition aboard the Mir Space Station can avoid the disadvantages mentioned above. The gravity level can also be changed by rotating the test stand with corresponding angular velocity. The working time deficit for the present space experiment aboard the Mir Space Station did not allow fulfilling the whole planned program. Therefore, only 10 experimental runs at $0.1 g$ and one at $0.014 g$ have been conducted. The limited number of experimental runs at the partial gravity conditions detracts from any meaningful interpretation of the data. In the present paper, only the experimental results at the background microgravity environment of the Mir Space Station (no more than $10^{-5} \mathrm{~g}$ ) are presented and analysed.

\section{Experimental facility and procedure}

The experimental facility, mounted in the "Volna-2A" stand aboard the Mir Space Station, is shown schematically in Fig. 1. The Volna-2A stand can be rotated on its axis with the corresponding angular velocity $\omega$, providing an "equivalent" low-gravity condition $g_{\text {equ }}=\omega^{2} R$ at the designed position at a distance $R$ from the rotary axis. Without rotation of the Volna-2A stand, the experiments were conducted at microgravity condition (no more than $10^{-5} \mathrm{~g}$ ), which is the background environment of the Mir Space Station.

The $10 \mathrm{~mm}$ bore circular test tube (E) is drilled in an organic glass prism with a $16 \times 16 \mathrm{~mm}^{2}$ square cross-section. The length of the test tube is $356 \mathrm{~mm}$, which is limited by the spatial restrictions of the Mir Space Station. The test tube is parallelized at a distance $620 \mathrm{~mm}$ from the

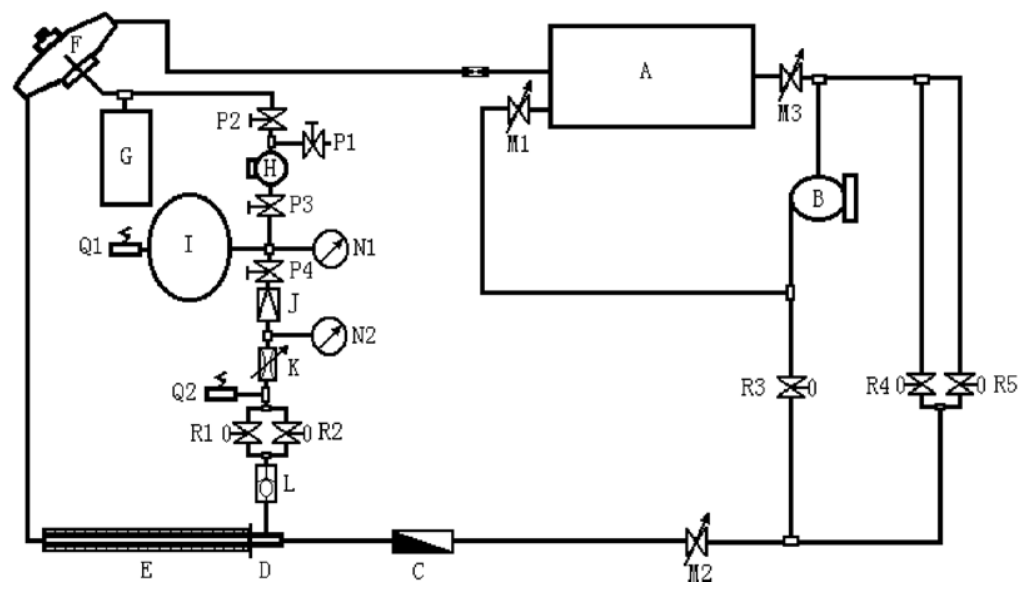

Fig. 1. Schematic of the experimental facility aboard the Mir Space Station. (A) Liquid storage vessel, (B) pumb, (C) turbine flowmeter, (D) mixer, (E) test tube, (F) separator, (G) rubber vessel, (H) compressor, (I) gas storage tank, (J) pressure regulator, (K) throttle for gas flow rate, (L) back valve, (M1-M3) throttle for liquid flow rate, (N1-N2) pressure readouts, (P1-P4) valves, (Q1-Q2) relief valves, (R1-R5) solenoid valves. 
rotary axis of the Volna-2A stand. Then it is horizontal with respect to the pseudo-gravitational force. As mentioned above, only the results of two-phase flow patterns at microgravity condition are reported and analysed in the present paper.

Air is used as the gas phase in the space experiments. Carbogal, an odourless, colourless, and non-toxic liquid with a small contact angle $\left(0^{\circ}-7^{\circ}\right)$ with the test tube, is used as the liquid phase. Its main characteristics are as follows: density of $1858 \mathrm{~kg} / \mathrm{m}^{3}$, viscosity of $1.05 \times 10^{-6} \mathrm{~m}^{2} / \mathrm{s}$, surface tension of $0.019 \mathrm{~N} / \mathrm{m}$. A certain volume of the gas and the liquid are operated in a closeloop system, in which no component leakage to the space station compartment is allowed. Before the beginning of the experiments, a part of air is compressed from the station compartment into a gas tank (I) in the system. The charged air is repeatedly used to carry out all the experimental runs. A pressure readout (N1) is used to measure the air pressure in the tank. After a pressure regulator (J), the air pressure is reduced to $0.15 \mathrm{MPa}$. A liquid vessel (A) in the system is used to store the whole supply of carbogal, which is also used repeatedly in the experiments. The two phases are supplied to the test tube through a Venturi mixer (D). Three groups of $0.75 \mathrm{~mm}$ diameter drillings, six drillings per group, are located at different cross-sections but uniformly around the periphery of the Venturi tube (Fig. 2). Carbogal is injected axially while air is introduced radially. After the test tube, the gas-liquid mixture enters a separator (F). Carbogal remains in the separator while air fills in a rubber vessel $(G)$. Several experimental runs can be conducted until either the separator is totally filled with carbogal or the decreased air pressure in the tank falls down to a specific value. Then carbogal is pumped from the separator to the storage vessel while air is compressed from the rubber vessel to the tank. Further experimental runs can be conducted again.

The liquid flow rates are set by three throttle valves (M1-M3), while the gas flow rates are adjusted by one throttle valve (K). Two turbine flowmeters (C) with different nominal ranges are used for the measurement of the liquid flow rates in the space experiments. A multimeter is used to measure the frequency outputs of the flowmeters. They were calibrated on Earth prior to delivery

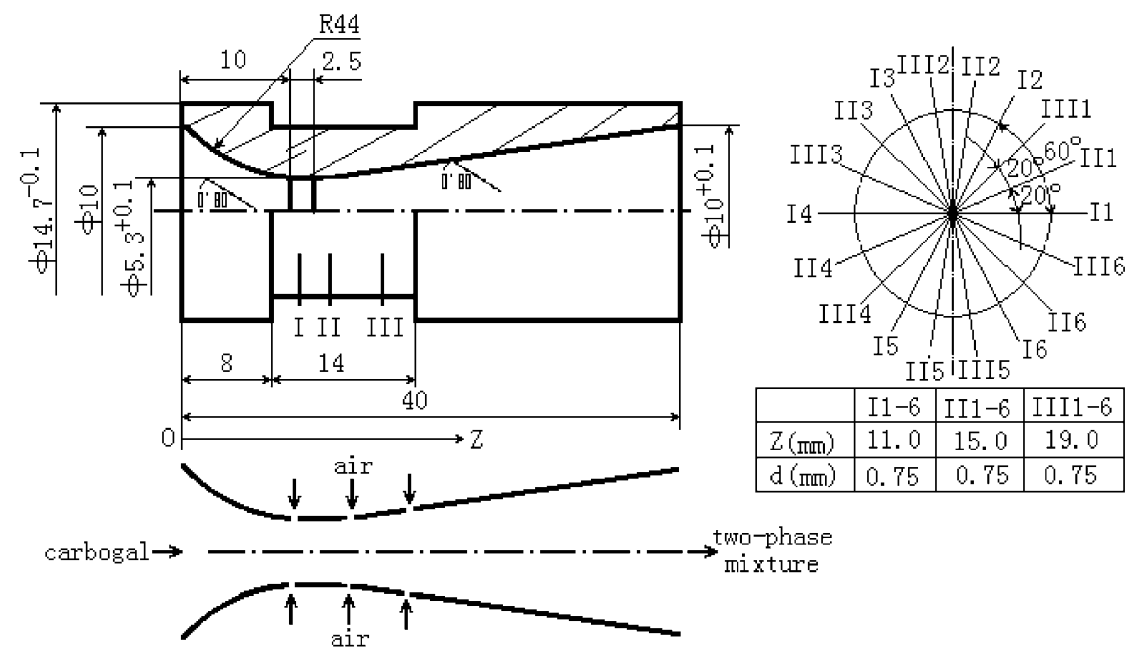

Fig. 2. Geometry of the gas-liquid mixer. 
to the Mir Space Station. The averaged gas flow rate over the measurement period is determined through the pressure drop in the tank, using the ideal gas law. It was shown in the calibration on Earth that the absolute pressure in the test tube is approximately equal to the atmospheric pressure when the rubber vessel is not full, and that the change of the temperature in the tank can be neglected for determining the used air amount. The uncertainties in the measurement of the gas and liquid superficial velocities are estimated to be respectively less than $20 \%$ and $15 \%$ over the ranges investigated in these studies.

The flow patterns in the second half of the test tube (longer than $150 \mathrm{~mm}$ ) were recorded by a digital video camera SONY DCR-VX1000 at standard video rate of about 30 images/s. The test tube was lit by two lamps of $40 \mathrm{~W}$, which are part of the lighting system of the Volna-2A stand. It has been shown in the ground experiments that the camera shutter speed of $1 / 2000 \mathrm{~s}$ is sufficient to eliminate image blur. Unfortunately, the video camera was incorrectly operated with the automatic mode by the cosmonaut-investigators, not with the designed shutter speed of 1/2000 s, during the space experiments. That results in some difficulties in the identifying of the flow patterns.

The multimeter, the pressure readouts $(\mathrm{N} 1, \mathrm{~N} 2)$, the experiment number counter and a part of the dial showing the head position of the throttle valve $(\mathrm{K})$ are also in the recorded region and then can be viewed in the video camera images. Thus the gas and liquid superficial velocities can be calculated based on the replay of the videotape in the laboratory on Earth.

\section{Flow pattern observations}

In the present space experiment, the gas and liquid superficial velocities were varied in the range of $0.09-6.3$ and $0.04-0.81 \mathrm{~m} / \mathrm{s}$, respectively. The information about the flow structure is arrived at by carefully observing a sequence of successive frames with replay of the videotape at standard speed, slowed speed, or image-by-image manner in the laboratory. A panel of observers analysed the videotape records. In some cases, there was not complete agreement among several observers in classifying the flows. The regime used in further analysis was determined by weight of evidence, namely by the greater number of observers in agreement or near agreement.

Some representative hand traces are displayed in Fig. 3 to convey the basic observations of the flow patterns at microgravity condition, which can be classified as five kinds:

(1) Dispersed bubble flow (Fig. 3(a)). It is featured by the presence of a large number of small bubbles with diameter of no more than $1 \mathrm{~mm}$. It is realized when the liquid superficial Reynolds number $\operatorname{Re}_{\mathrm{SL}}=\rho_{\mathrm{L}} U_{\mathrm{SL}} D / \mu_{\mathrm{L}}$ is greater than 4000 .

(2) Bubble flow (Fig. 3(b)). In this (ordinary) bubble flow regime, the size of gas bubbles is in the range of $2-8 \mathrm{~mm}$. Bubbles with different diameters move at approximately the same velocity, showing that the local velocity slip between the two phases is small at microgravity.

(3) Slug flow (Fig. 3(c)). Elongated gas bubbles (so-called Taylor bubbles) having regular hemispherical nose shapes occur in the test tube. The flow structure is essentially axisymmetrical. The length of the Taylor bubbles does not exceed the observation section. For the Taylor bubble with length of 3-4 diameters of the test tube, not only the nose but also the rear has a shape close to hemispherical. The rear of longer Taylor bubbles may lose the hemispherical shape. 
(a)

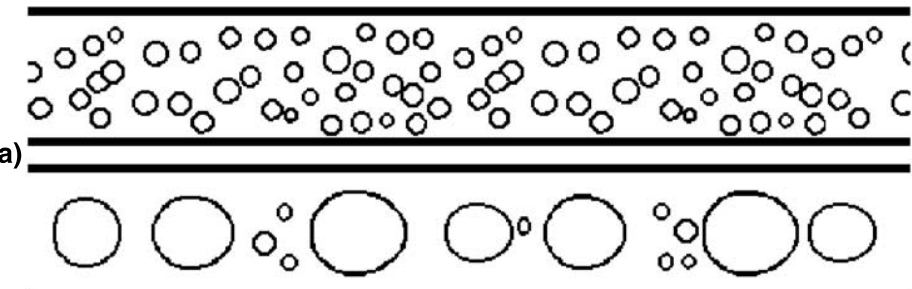

(b)

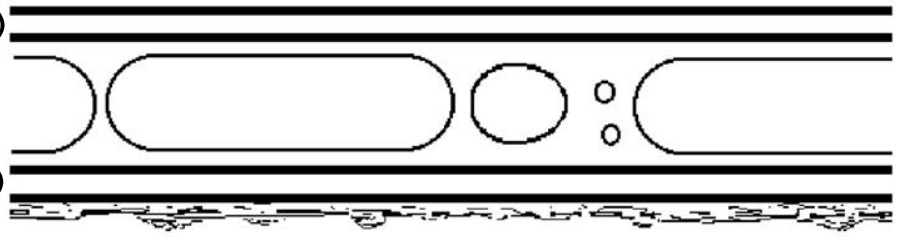

(d)

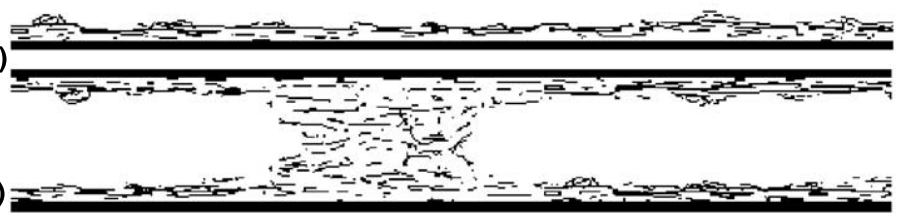

Fig. 3. Characteristics of the flow patterns observed at microgravity condition. (a) Dispersed bubble flow, (b) bubble flow, (c) slug flow, (d) annular flow, (e) slug-annular transitional flow.

(4) Annular flow (Fig. 3(d)). It is observed when the gas phase flows uninterruptedly at the center of the test tube and the liquid phase flows in a wavy film at the wall.

(5) Slug-annular transitional flow (Fig. 3(e)). It is transitional flow between slug flow and annular flow. The liquid flows as a film continuously along the wall of the test tube. However, there exist irregularly some frothy structures blocking the whole test tube. The distance between two adjacent frothy structures often exceeds the length of the observation section.

It ought to be noted here that, due to the image blur and insufficient resolutions caused by the insufficiency of the camera shutter speed used incorrectly in the space experiments, some data of slug or annular flows, especially slug flows at high velocity, may be included in the last category. Therefore, in the following comparison, the slug flow and the slug-annular transitional flow are all regarded as intermittent flows. The dispersed bubble flow and the bubble flow are also regarded as one category, namely bubble flow, in the following analysis.

\section{Analysis of experimental data}

As mentioned above, the length-to-diameter ratio of the test tube is only 36 in the present space experiment. Thus the observed flow patterns should be considered to be developing ones. In the literature, however, the great majority of efforts are devoted to fully developed two-phase flow at microgravity condition. Some widely used models have been developed based on the analysis of fully developed two-phase flows at microgravity condition. The model proposed by Lee (1993), as far as the authors know, is the sole one that accounts for the entrance effect on the flow pattern 
transitions at microgravity condition. It is then reasonable to compare the present experimental data with the predictions of Lee's model and some other models in order to make evaluations of these models and to have some insights on the flow evolution.

According to Lee (1993), there are different mechanisms controlling the flow pattern transitions in three different domains, namely "low", "middle" and "high" gas flow rate domains. The boundaries between two adjacent domains lie respectively in

$$
\frac{\rho_{\mathrm{G}}\left(U_{\mathrm{SG}} A_{\mathrm{t}} / n\right)^{2} / A_{\mathrm{n}}}{\pi d_{\mathrm{n}} \sigma f(\varphi)}=0.1 \text { and } 10,
$$

where $A_{\mathrm{t}}$ is the tube cross-section area, $n, A_{\mathrm{n}}$ and $d_{\mathrm{n}}$ are, respectively, the number, the cross-section area and the diameter of the gas injector nozzles, $f(\varphi)$ is a function of the inclination angle between the nozzle and the tube. According to $\operatorname{Kim}(1992)$, the value of $f(\varphi)=0.9$ is taken for the cross-flow multi-nozzle system as used in the present study.

The detailed transition criteria in the model proposed by Lee (1993), which will be given in the following subsections, are derived by scaling analysis of the related forces in the formation of bubbles at the tube inlet and considering the possible evolution of the inlet region flow structure in the downstream region. Recent studies on bubble formation at microgravity condition are presented by Bhunia et al. (1998), Pais (1999), and Nahra and Kamotani (2000), among other investigators. They judiciously pointed out that the difference between the liquid velocity and the gas injection velocity can either enhance or retard bubble departure from the injection site and can then affect the flow evolution downward. It ought to be pointed out here that, in the transition criteria proposed by Lee (1993) which correspond to the present experimental conditions, this influence has also been taken into account. The influence of the gas injection velocity on the viscous drag force acting upon the gas bubble is neglected only in the "low" gas flow rate domain, where the gas injection velocity is so small that the neglect of its effect is reasonable.

\subsection{Bubble-to-intermittent transition}

According to Lee (1993), the bubble-to-intermittent transition occurs in the "low" and "middle" gas flow rate domains. In the "low" gas flow rate domain, the bubble-to-slug transition is controlled by the surface tension and the viscous drag forces. It occurs at

$$
U_{\mathrm{SL}}=\sqrt{\frac{8 \sigma d_{\mathrm{n}}}{C_{\mathrm{D}} \rho_{\mathrm{L}} D^{2}}},
$$

where $C_{\mathrm{D}}$ is the drag coefficient. According to Moore (1965) and Maxworthy et al. (1996), the curve of the drag coefficient of a gas bubble against the bubble Reynolds number $\operatorname{Re}_{\mathrm{B}}=U_{\mathrm{B}} D_{\mathrm{B}} / v_{\mathrm{L}}$ is dependent upon the Morton number $M o=g v_{\mathrm{L}}^{4} \rho_{\mathrm{L}}^{3} / \sigma^{3}$ which has a value of $M o \approx 10^{-13}$ in the present experiment. It is assumed that at the bubble-to-intermittent transition the size of gas bubble formed near the gas injector is approximate to the diameter of the throat of the Venturi mixer $(5.3 \mathrm{~mm})$. The relative velocity between gas bubble and liquid flow in the "low" gas flow rate domain may have an order of $0.1 \mathrm{~m} / \mathrm{s}$. Therefore, the bubble Reynolds number is approximate to 500, and the value of $C_{\mathrm{D}} \approx 0.135$ will be taken according to the experiments of Maxworthy et al. (1996) and the theory of Moore (1965). A constant liquid superficial velocity is 
predicted for the transition, which has succeeded in distinguishing between the bubble flow and the slug flow in the "low" gas flow rate domain of the present experiment (Fig. 4).

In the "middle" gas flow rate domain, when

$$
\frac{U_{\mathrm{SG}} / n}{U_{\mathrm{SL}}} \leqslant 0.025 C_{\mathrm{D}} \frac{\rho_{\mathrm{L}}}{\rho_{\mathrm{G}}} \frac{A_{\mathrm{n}}}{A_{\mathrm{t}}},
$$

and

$$
W e_{\mathrm{SL}} \leqslant 870 \sqrt{U_{\mathrm{SL}} /\left(U_{\mathrm{SG}} / n\right)} R_{\mathrm{SL}}^{1 / 5}
$$

just as in the present case, the flow pattern transitions are controlled by the initial size of gas bubbles formed near the tube inlet and the coalescence between the bubbles in the downstream region. According to Lee (1993), the bubble-to-slug transition will occur at

$$
\sqrt{U_{\mathrm{SL}} /\left(U_{\mathrm{SG}} / n\right)}=C \gg 0.4,
$$

where $C$ is an empirical parameter. If the value of the parameter $C$ is taken as 2.4, Eq. (5) can give a very satisfied agreement with the experimental data (Fig. 4).

The most widely used model for the bubble-to-slug transition in fully developed two-phase flows at microgravity is the drift-flux model, or void fraction model (Dukler et al., 1988; Colin et al., 1991, 1996; Bousman et al., 1996). According to this model, the basic mechanism that controls the transition from bubble to slug flow is the coalescence of bubbles. The rate of coalescence depends upon the number of collisions, which is related to the bubble packing or void fraction $\varepsilon$. Thus the bubble-to-slug transition will be expected to occur at a critical void fraction $\varepsilon_{\mathrm{cr}}$. Due to the difference between the radial profiles of void fraction and velocities, there is an average drift in two-phase flows even if there is no local slip (Colin et al., 1996):

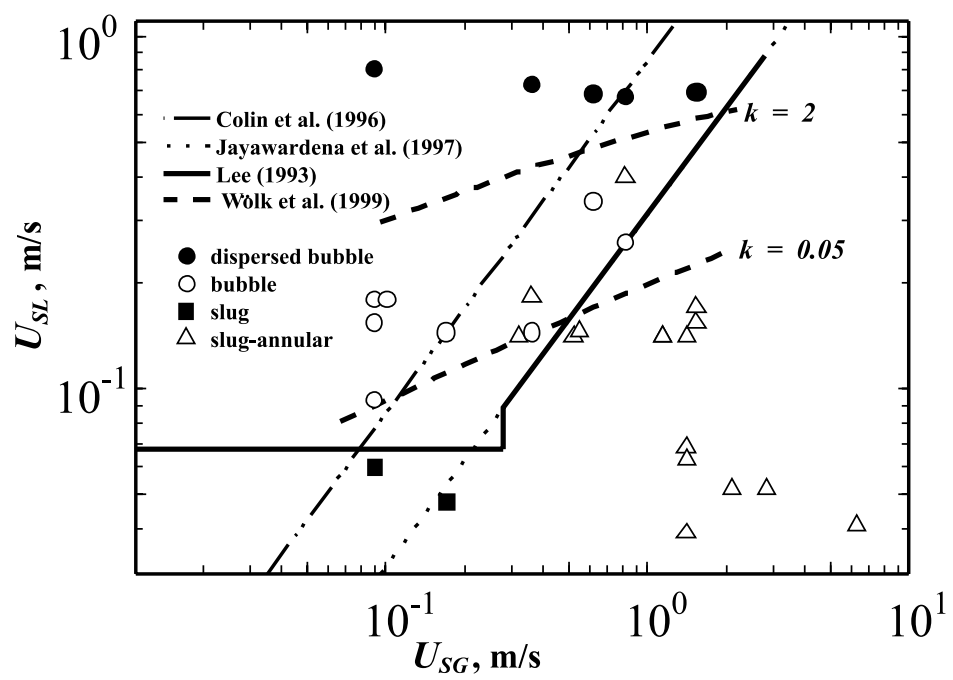

Fig. 4. Bubble-to-intermittent transition at microgravity condition. 


$$
U_{\mathrm{SL}}=\frac{1-C_{0} \varepsilon}{C_{0} \varepsilon} U_{\mathrm{SG}}
$$

where $C_{0}$ is the gas phase distribution parameter. Substituting $\varepsilon_{\mathrm{cr}}$ for $\varepsilon$ in the above equation, the bubble-to-slug transition may be expressed as

$$
U_{\mathrm{SL}}=\frac{1-C_{0} \varepsilon_{\mathrm{cr}}}{C_{0} \varepsilon_{\mathrm{cr}}} U_{\mathrm{SG}}
$$

Let $X_{C}=C_{0} \varepsilon_{\mathrm{cr}}$. Colin et al. (1996) proposed an empirical relationship as

$$
\begin{array}{ll}
X_{C} \approx 0.54, & \text { for } S u<1.5 \times 10^{6} ; \\
X_{C} \approx 0.24, & \text { for } S u<1.7 \times 10^{6} .
\end{array}
$$

In the above equations, $S u=\rho_{\mathrm{L}} \sigma D / \mu_{\mathrm{L}}^{2}$ is the Suratman number. An alternative relationship can be obtained by rearranging the empirical bubble-to-slug transition criterion proposed by Jayawardena et al. (1997), which reads as

$$
X_{C}=K_{1} \frac{v_{\mathrm{G}}}{v_{\mathrm{L}}} /\left(K_{1} \frac{v_{\mathrm{G}}}{v_{\mathrm{L}}}+S u^{2 / 3}\right),
$$

where $K_{1}=464.16$ is an empirical constant. It ought to be noted that the above relations are extracted from nearly the same experimental database, in which the Suratman number is in the range approximately from $10^{4}$ to $10^{6}$ and the critical void fraction for transition approximately from 0.1 to 0.5 .

In the present experiments, the Suratman number $S u \approx 9.28 \times 10^{4}$. The void fraction was not measured in this experiment. It is, however, reasonable to assume that the above empirical relationships can be used in the present study since the value of the Suratman number is in the range of validity. Then the transitional quality $X_{C} \approx 0.54$ according to Eq. (8a), while $X_{C} \approx 0.76$ according to Eq. (9). It is clearly shown in Fig. 4 that the predictions of the drift-flux model with these two values approximately give the upper and lower boundaries of the bubble-to-intermittent transition. However, the transitional lines predicted with these values always have slopes steeper than that observed and no satisfactory agreement can be obtained for the whole range. If the data of low gas flow rates are not considered, the prediction of the drift-flux model with the value of $X_{C} \approx 0.76$ can give a more satisfactory agreement with the experimental data, which is also coincident with that of Lee's model. In fact, Eq. (5) can be rewritten in the same form as the driftflux model and the transitional quality $X_{C}=\left(1+C^{2} / n\right)^{-1}$.

It can be concluded, based on the above comparison, that the flow patterns in the case of low gas flow rate should be considered to be developing due to the relatively small length-to-diameter ratio of the test tube used in the present study. For the case of middle gas flow rate, Lee's model is equivalent to the drift-flux model with the value of the transitional quality $X_{C}$ predicted by Eq. (5), and can give a more satisfactory agreement with the experimental data. It may be indicated that the entrance effect could be neglected for such flows.

Recently, based on their experimental investigations of two-phase flow in small-diameter channels at normal- and low-gravity conditions, Wölk et al. (1999) found that satisfactory agreements between the experimental results and the predictions of the drift-flux model can be obtained only for the "middle" liquid flow rate domain. The authors proposed a transition 
criterion for the "low" liquid flow rate at microgravity condition based on the balance between the "minimum" total free energy flux and the total turbulent kinetic energy. It reads as

$$
\frac{U_{\mathrm{SL}}}{U_{\mathrm{SG}}} \frac{W e_{\mathrm{L}}}{R e_{\mathrm{L}}^{n}}=\frac{8}{3} \frac{k}{C_{\mathrm{T}}},
$$

where $W e_{\mathrm{L}}=\rho_{\mathrm{L}} U_{\mathrm{L}}^{2} D / \sigma$ and $R e_{\mathrm{L}}=U_{\mathrm{L}} D / \mu_{\mathrm{L}}$ are the liquid Weber number and the liquid Reynolds number, respectively. $C_{\mathrm{T}}$ and $n$ are defined in the Blasius correlation for the friction coefficient in turbulent pipe flow, i.e. $f=C_{\mathrm{T}} R e_{\mathrm{L}}^{-n}$, and for example $C_{\mathrm{T}}=0.079$ and $n=0.25$ for the circular tube. The parameter $k$ is an empirical constant. Wölk et al. (1999) found that Eq. (10) with $k=2$ for the circular tube and $k=0.8$ for the rhombic channel can predict the transition well for their experiments with equivalent (hydraulic) diameter of $6 \mathrm{~mm}$. The authors argued that the different values of $k$ are caused by the influence of the turbulent secondary flow.

It is shown by comparison of the liquid superficial velocities between the present experiment and those of Wölk et al. (1999) that the bubble-to-intermittent transition in the present study is in the "low" liquid flow rate domain. The prediction of Eq. (10) is also plotted in Fig. 4. It is shown that the predicted transition boundary with the value of $k=2$ lies between the dispersed bubble and the (ordinary) bubble flows in the present case. Only when a much smaller value of $k=0.05$ is taken can the transition from bubble to intermittent flow be predicted well. This means that the value of $k$ may be extremely dependent upon the flow conditions. This will extremely restrict its application because of the complexity of two-phase turbulent flows.

\subsection{Intermittent-to-annular transition}

As suggested by Lee (1993), annular flow will occur when

$$
\frac{U_{\mathrm{SL}}}{U_{\mathrm{SG}} / n} \ll \frac{A_{\mathrm{t}}}{A_{\mathrm{n}}}
$$

in the "high" gas flow rate domain. Under the above condition, the kinetic force of the gas is sufficient to push the gas bubble forward before it becomes large. As a result, the gas flow is no longer a stream of bubbles, but is instead a jet flow. Therefore, annular flow is expected. This is just the case in the present experiment. The transitional boundary between intermittent and annular flows is then just the line corresponding to Eq. (1), in which the left-side term should take the value of 10. As shown in Fig. 5, it agrees the present experimental data very well.

Recently, Zhao and $\mathrm{Hu}$ (2000) proposed a semi-theoretical Weber number model about the transition from slug to annular flow at microgravity condition. It assumed that the basic mechanism controlling the transition from slug to ordinary annular is the balance between the impulsive force due to the gas inertia and the surface tension force. Taking into account the relative motion between the gas and liquid phases, the impulsive force due to the gas inertia can be expressed as $\pi R_{\mathrm{t}}^{2} \rho_{\mathrm{G}} U_{\mathrm{SG}}\left(U_{\mathrm{G}}-U_{\mathrm{L}}\right)$, where $U_{\mathrm{G}}=U_{\mathrm{SG}} / \varepsilon, U_{\mathrm{L}}=U_{\mathrm{SL}} /(1-\varepsilon)$ and $R_{\mathrm{t}}$ are, respectively, the gas averaged velocity, the liquid averaged velocity and the inside radius of the test tube. The surface tension force acted on the gas-liquid interface is expressed as $2 \pi R_{\mathrm{B}} \sigma$, where $R_{\mathrm{B}}$ is the radius of the gas core or that of the nose of the elongated gas bubble near the transition. With the use of the drift-flux relationship, the transition may be expressed as 


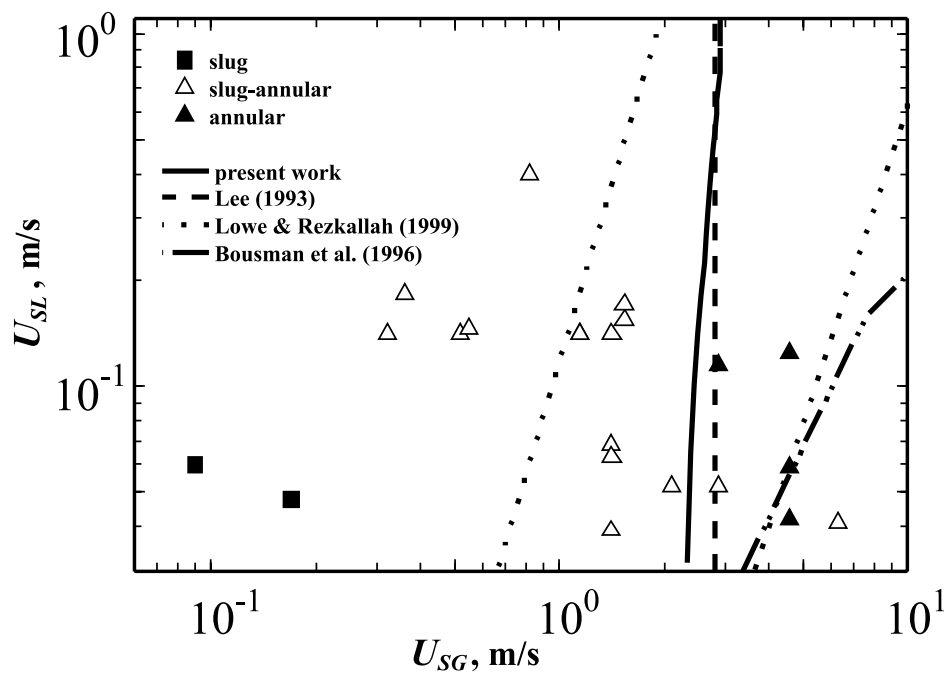

Fig. 5. Intermittent-to-annular transition at microgravity condition.

$$
\begin{aligned}
& U_{\mathrm{SG}}=\sqrt{\frac{4 \kappa C_{0} \sigma \varepsilon^{3 / 2}(1-\varepsilon)}{\left(C_{0}-1\right) \rho_{\mathrm{G}} D}}, \\
& U_{\mathrm{SL}}=\frac{1-C_{0} \varepsilon}{C_{0} \varepsilon} U_{\mathrm{SG}}
\end{aligned}
$$

where $\kappa$ is an empirical parameter of an order of 1 . Using the definitions of the gas and liquid superficial Weber numbers $W e_{\mathrm{SK}}=\rho_{\mathrm{K}} U_{\mathrm{SK}}^{2} D / \sigma$ (where the subscript $\mathrm{K}=\mathrm{G}$ or $\mathrm{L}$ ), the above equations can be rewritten in dimensionless form, which is the reason for its name.

The above equations are slightly different from those proposed by Zhao and $\mathrm{Hu}(2000)$ due to the modification of the expressions of the impulsive and surface tension forces. It does not cause obvious difference of the predictions, but a more physical base will be obtained.

Now the value of $\kappa=1$ is taken in the above equations, while $C_{0}=1.16$ is taken for the twophase flow in circular tube at microgravity, as suggested by Zhao and $\mathrm{Hu}$ (2000). It is shown in Fig. 5 that the transition from intermittent to annular flow is predicted with good satisfaction by the present model. It is also shown that the boundaries of the transition predicted by the present model and Lee's model are close to each other, which indicates that such flows might be close to fully developed and the entrance effect may be neglected.

There is also an empirical Weber number model developed by Rezkallah and his colleagues (Zhao and Rezkallah, 1993; Rezkallah and Zhao, 1995; Rezkallah, 1996; Lowe and Rezkallah, 1999), in which the slug-annular transitional flow is regarded as a basic regime. It is, however, pointed out by Zhao and $\mathrm{Hu}$ (2000) that the regime of slug-annular transitional flow predicted by this model is often too wide to contain some annular, slug and bubble flow data. Its latest transitional lines, proposed by Lowe and Rezkallah (1999), are also plotted in Fig. 5. It is shown that, although the boundary between slug-annular transitional flow and slug or bubble flow can be predicted well, this model cannot predict well the boundary between slug-annular transitional flow and annular flow in the present experiment. 
Along another approach, Dukler et al. (1988) and later Bousman et al. (1996) proposed a void fraction matching slug-to-annular transition model. A force balance approach is used to determine a void fraction relationship for annular flow and then it is equated with a void fraction expression developed from the drift-flux model. Fig. 5 also shows the prediction of this model, which is much less successful in predicting the intermittent-to-annular transition in the present experiment.

\section{Conclusion}

A series of experiments on two-phase gas-liquid flow patterns in a test tube with a length of 356 $\mathrm{mm}$ and an inside diameter of $10 \mathrm{~mm}$ were conducted aboard the Mir Space Station in August 1999. A new set of experimental flow pattern data at the background microgravity environment of the Mir Space Station (no more than $10^{-5} \mathrm{~g}$ ) are reported. Five kinds of flow patterns, namely dispersed bubble flow, bubble flow, slug flow, slug-annular transitional flow, and annular flow, were observed in the space experiment. Due to the small length-to-diameter ratio of the test tube used in the present study, the observed flow patterns should be considered to be developing ones. The experimental results are compared with the model proposed by Lee (1993), which accounts for the entrance effects on the flow pattern transitions. A good agreement between the predictions and the experimental data was obtained.

Some widely used models developed based on the analysis of fully developed two-phase flow at microgravity condition are also compared with the present data in order to make evaluations of these models and to have some insights on the flow evolution. It is found that a semi-theoretical Weber number model, initially proposed by Zhao and $\mathrm{Hu}$ (2000) and improved in the present study, can predict better the transition to annular flow. The empirical Weber number model developed by Rezkallah and his colleagues (Zhao and Rezkallah, 1993; Rezkallah and Zhao,

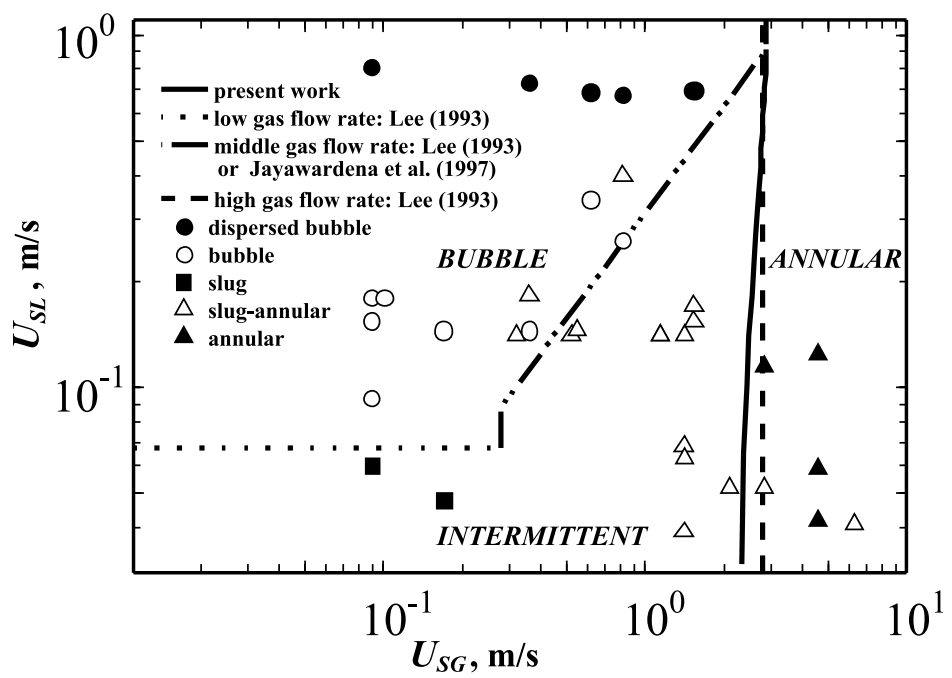

Fig. 6. Comprehensive flow pattern map with the best predictions for each transition. 
1995; Rezkallah, 1996; Lowe and Rezkallah, 1999) may overestimate the gas superficial velocity at the boundary between annular and slug-annular transitional flows. The widely used drift-flux model cannot predict well the bubble-to-intermittent transition observed in the present experiment; neither can the model proposed by Wölk et al. (1999).

The differences between the predictions of Lee's model and other models are quite clear in the case of low gas flow rate. It may be indicated that the two-phase flows are still developing due to the short length-to-diameter ratio of the test tube used in the present study. For the cases of middle and high gas flow rate, however, the predictions of Lee's model are very close to that of the drift-flux model with the value of transitional quality determined by Jayawardena et al. (1997) and that of the semi-theoretical Weber number model, respectively. It is indicated that such flows might be close to fully developed and the entrance effect may be neglected.

Based on the above conclusions, a comprehensive flow pattern map with the best predictions for each transition is provided in Fig. 6.

It ought to be pointed out that, due to the camera shutter speed being insufficient in the present study, there could be a wide range of interpretations on the imaging results, especially with regard to defining the boundary between slug and annular flow. Further work, therefore, is required with sufficient resolution of images in order to sharpen up the behaviour, or position, of the transition lines.

\section{Acknowledgements}

This work is supported by the grant 19789201 of the National Natural Science Foundation of China, the grant "95-Yu-34" of the Ministry of Science and Technology of China, and the Postdoctoral Science Foundation of China. The space experiment was performed by the collaboration with the Keldysh Research Center of Russia. The authors wish to express their gratitude to the cosmonauts for their tremendous efforts.

\section{References}

Bhunia, A., Pais, S., Kamotani, Y., Kim, I., 1998. Bubble formation in a coflow configuration in normal and reduced gravity. AIChE J. 44, 1499-1508.

Bousman, W.S., McQuillen, J.B., Witte, L.C., 1996. Gas-liquid flow patterns in microgravity: effects of tube diameter, liquid viscosity and surface tension. Int. J. Multiphase Flow 22, 1035-1053.

Carron, I., Best, F.R., 1994. Gas-liquid annular flow under microgravity conditions: a temporal linear stability study. Int. J. Multiphase Flow 20, 1085-1093.

Carron, I., Best, F.R., 1996. Microgravity gas/liquid flow regime maps: can we compute them from first principles? In: Heat Transfer - Houston 1996 AIChE Symposium Series, 92, no. 310, pp. 92-99.

Colin, C., Fabre, J., Dukler, A.E., 1991. Gas-liquid flow at microgravity conditions - I. Dispersed bubble and slug flow. Int. J. Multiphase Flow 17, 533-544.

Colin, C., Fabre, J., McQuillen, J., 1996. Bubble and slug flow at microgravity conditions: state of knowledge and open questions. Chem. Eng. Comm. 141-142, 155-173.

Dukler, A.E., Fabre, J.A., McQuillen, J.B., Vernon, R., 1988. Gas-liquid flow at microgravity conditions: flow patterns and their transitions. Int. J. Multiphase Flow 14, 389-400.

Galbiati, L., Andreini, P., 1994. Flow pattern transition for horizontal air-water flow in capillary tubes. A microgravity “equivalent system" simulation. Int. Commun. Heat Mass Transfer 21, 461-468. 
Jayawardena, S.S., Balakotaiah, V., Witte, L.C., 1997. Flow pattern transition maps for microgravity two-phase flows. AIChE J. 43, 1637-1640.

Kim, I.W., 1992. Modelling of bubble and drop formation in flowing liquids in microgravity. Ph.D thesis, Case Western Reserve University.

Lee, D., 1987. Thermohydraulic and flow regime analysis for condensing two-phase flow in a microgravity environment. Ph.D. thesis, Texas A \& M University.

Lee, J., 1993. Scaling analysis of two-phase gas-liquid flow pattern in microgravity. In: 31st Aerospace Sciences Meeting \& Exhibit, January 11-14, Reno, NV.

Lovell, T.W., 1985. Liquid-vapor flow regime transitions for use in design of heat transfer loops in spacecraft. AFWAL-TR-85-3021.

Lowe, D.C., Rezkallah, K.S., 1999. Flow regime identification in microgravity two-phase flows using void fraction signals. Int. J. Multiphase Flow 25, 433-457.

Maxworthy, T., Gnann, C., Kürten, M., Durst, F., 1996. Experiments on the rise of air bubbles in clean viscous liquids. J. Fluid Mech. 321, 421-441.

Moore, D.W., 1965. The velocity of rise of distorted gas bubbles in a liquid of small viscosity. J. Fluid Mech. 23, 744 766.

Nahra, H.K., Kamotani, Y., 2000. Bubble formation from wall orifice in liquid cross-flow under low gravity. Chem. Eng. Sci. 55, 4653-4665.

Pais, S.C., 1999. Bubble generation in a continuous liquid flow under reduced gravity conditions. NASA CR 1999209170.

Reinarts, T.R., 1995. Slug to annular flow regime transition modeling for two-phase flow in a zero gravity environment. In: 30th International Energy Conversion Engineering Conference, July 30-August 4, Orlando, FL.

Reinarts, T.R., Ungar, E.K., Butler, C.D., 1995. Adiabatic two-phase pressure drop in microgravity: TEMP2A-3 flight experiment measurements and comparison with predictions. In: 33rd Aerospace Sciences Meeting \& Exhibit, January 9-12, Reno, NV.

Rezkallah, K.S., 1996. Weber number based flow-pattern maps for liquid-gas flows at microgravity. Int. J. Multiphase Flow 22, 1265-1270.

Rezkallah, K.S., Zhao, L., 1995. A flow pattern map for two-phase liquid-gas flows under reduced gravity conditions. Adv. Space Res. 16, 133-136.

Wölk, G., Dreyer, M., Rath, H.J., 1999. Investigation on two-phase flow in small diameter non-circular channels under low and normal gravity. In: Space Technology \& Applications International Forum - 1999, Albuquerque, NM. AIP-Conference 458, pp. S785-S791.

Zhao, J.F., 1999. A review of two-phase gas-liquid flow patterns under microgravity conditions. Adv. Mech. 29, 369382 (in Chinese).

Zhao, J.F., Hu, W.R., 2000. Slug to annular flow transition of microgravity two-phase flow. Int. J. Multiphase Flow 26, $1295-1304$.

Zhao, J.F., Lin, H., Xie, J.C., Hu, W.R., 2000. Experimental investigation of two-phase gas-liquid flow at reduced gravity. In: 4th Global Chinese Symposium on Space Science \& Technology, April 11-14, Taiwan, China (in Chinese).

Zhao, L., Rezkallah, K.S., 1993. Gas-liquid flow patterns at microgravity conditions. Int. J. Multiphase Flow 19, 751763.

Zhao, L., Rezkallah, K.S., 1995. Pressure drop in gas-liquid flow at microgravity conditions. Int. J. Multiphase Flow 21, 837-849. 